\title{
Editorial
}

\section{A welcome to the CMP edition}

The last decade has witnessed great forward strides in the field of molecular medicine. Unfortunately, the term "molecular medicine" is often applied or interpreted in too narrow a fashion, in that it has been taken to imply only those research results involving DNA technology. We consider this to be erroneous, as "molecular" is clearly a word with wider implications. Thus, methods of molecular genetics, including DNA and RNA hybridisation and the polymerase chain reaction are only part of a wider spectrum of approaches for investigating the mechanisms and effects of disease processes. Other methodologies encompassing "molecular medicine" include-for example, ion probes for the study of cytoplasmic events, hybridoma technology, histochemistry, ultrastructural studies, and protein and enzyme biochemistry; indeed, any approach investigating the properties of tissues, cells and their constituents. For instance, the development by Kohler and Milstein of the methods for producing monoclonal antibodies resulted from an understanding of fundamentals of cell physiology. This has had far-reaching effects on basic research, diagnostic pathology and on new approaches to the treatment of diseases.

The importance of progress in research and diagnosis is relevant to all of the subdisciplines of pathology and this is the raison d'être for the Clinical Molecular Pathology (CMP) edition of the fournal of Clinical Pathology (FCP). We feel that the strength of the CMP edition lies in its multidisciplinary approach since its contents will be relevant ultimately to all branches of pathology. This is reflected by the breadth of interests of the international editorial panel, who represent not only diagnosticians in the fields of pathology, but also other clinicians and scientists whose specialties, amongst others, include immunology, biochemistry, cell biology, oncology, microbiology, and genetics.

Why, then, this new publication? The approach of clinicians to the diagnosis and treatment of diseases is ultimately based on fundamental knowledge of the causes and consequences of pathological processes. An example of the bringing together of different disciplines in pathology lies in the marriage of virology and histopathology in the study of papilloma viruses and cervical dysplasia and of Epstein-Barr virus and Hodgkin's disease. Such investigations have used both the polymerase chain reaction and in situ DNA hybridisation techniques and have served to cast light on the causes of these conditions. Such un- derstanding of fundamental disease processes is frequently achieved through the recognition of observations and data which, at the outset, were not expected to have any particular applications to an area of medicine. It is often because of the insight, or luck, of an individual to recognise the potential of reports from unrelated fields, to make connections, that leads to a novel approach to a medical problem. Most investigators would acknowledge the value of browsing through the journals on the medical library shelf in the process of "creating" new ideas. Unfortunately, few have sufficient time to devote to this procedure; those library shelves are stacked with a multitude of journals containing potentially useful ideas from which "connections" could be made. Many of these journals are largely concerned with specialist areas of medicine or are basic science publications, each covering a vast range of esoteric topics. This rewarding but laborious process of "random" browsing is one for which selective searching through indexing systems is no substitute. Hence, the inception of the CMP edition. The aim is to provide a multidisciplinary journal which will be a vehicle for reporting the results of studies, using human material, which may be relevant to the causes and consequences of pathological processes. Thus the emphasis will be different from that of $\mathcal{F C P}$, which will continue to have an approach which is more "applied". Inevitably there will be a degree of overlap in the nature of the contents of $\mathscr{F C P}$ and $C M P$; this is intended, for we wish that the two publications will complement and supplement each other. A further advantage of this arrangement is that, despite being a new publication, the contents of CMP will be included immediately in the various indexing services. This should allay the fears of potential authors who are reluctant to submit manuscripts to a new publication for fear that the paper will be "lost".

The CMP edition will publish reviews, research papers, short reports, technical reports, and book reviews. We also encourage correspondence relating to published material.

It is timely that the $C M P$ edition is to begin publication during the silver jubilee year of $\mathcal{F C P}$. The publication team of $\mathcal{F C P}$ are justifiably proud to celebrate the contribution that $\mathcal{F C P}$ has made to the dissemination of knowledge in the field of pathology. It is also an opportune time to reflect on the future; hence, the birth of a new publication to carry on the tradition.

$\Re C$ and $D B$ 